\title{
HÁBITOS ALIMENTARIOS DE ANOURA GEOFFROYI (CHIROPTERA: PHYLLOSTOMIDAE) EN IXTAPAN DEL ORO, ESTADO DE MÉXICO
}

\author{
Luis Antonio CABALlero-MARTíneZ1, Irma Victoria Rivas MaNZaNo² \\ y Luis Isaac AGUILERA GóMEZ3 \\ Laboratorio de Ecología de la Facultad de Ciencias de la Universidad Autónoma del Estado de \\ México, Unidad Académica el Cerrillo, Piedras Blancas, Carretera Toluca - Atlacomulco Km. 15, \\ Desviación a Tlachaloya Km 3.5 Toluca, MÉXICO \\ 1caballero.1a@gmail.com; 2 victoriarivas60@yahoo.com.mx; 3luishalc@lycos.com
}

Caballero-Martínez, L. A., I. V. Rivas Manzano y L. I. Aguilera Gómez. 2009. Hábitos alimentarios de Anoura geoffroyi (Chiroptera: Phyllostomidae) en Ixtapan del Oro, Estado de México. Acta Zool. Mex. (n. s.) 5(1): 161-175.

RESUMEN. Se describen los hábitos alimentarios del murciélago Anoura geoffroyi en la Selva Baja Caducifolia de Ixtapan del Oro, Estado de México, para ello se colectaron quincenalmente durante un año muestras de residuos del pelaje y heces de 153 individuos. Se observó sólo granos de polen y restos de insectos en las muestras. Los morfotipos de polen encontrados corresponden a plantas que son frecuentadas por murciélagos nectarívoros, destaca por su frecuencia el género Ipomoea y se reporta por primera vez la presencia de Brugmansia candida, Stenocereus sp. y una especie de la familia Asteraceae en la dieta de $A$. geoffroyi. Las plantas determinadas por los tipos polínicos presentan características del síndrome quiropterofílico, sin embargo, también son polinizadas por mariposas nocturnas, por lo que considerando las adaptaciones morfológicas de $A$. geoffroyi para el consumo de néctar y que el $95 \%$ de los restos de insectos encontrados corresponden a lepidópteros, se podría catalogar a la especie en el sitio de estudio como un murciélago nectarívoro que presenta también la capacidad de alimentarse de lepidópteros asociados a las flores que frecuenta.

Palabras Clave: hábitos alimentarios, insectos, México, murciélagos, polen

Caballero-Martínez, L. A., I. V. Rivas Manzano \& L. I. Aguilera Gómez. 2009. Feeding habits of Anoura geoffroyi (Chiroptera: Phyllostomidae) in Ixtapan del Oro, Mexico State. Acta Zool. Mex. (n. s.) 5(1): 161-175.

ABSTRACT. We describe the feeding habits of the bat Anoura geoffroyi in the Seasonal Dry Tropical Forest at Ixtapan del Oro, Estado de Mexico. During a year, hair remainders and fecal samples from 153 individuals were collected every two weeks. We found just pollen grains and insect's debris in the samples. The pollen morphotypes correspond to plants that are frequently visited for nectar-feeding bats, the genus Ipomoea outstands for its frequency and it is the first report of Brugmansia candida, Stenocereus sp. and a species from Asteraceae family in the A. geoffroyi diet. The plants determined from pollen morphotypes present chiropterophilous syndrome characteristics, nevertheless, they are also pollinated by moths. Reason why and considering the morphological adaptations of $A$. geoffroyi for nectar consumption and the fact that $95 \%$ of the insects debris found in the samples correspond to lepidopterons, we could consider the species in the study place like a 
nectar-feeding bat that also has the capacity to eat lepidopterons associated to the flowers where this bat forages.

Key words: Bats, feeding habits, insects, Mexico, pollen

\section{INTRODUCCIÓN}

Los murciélagos de la familia Phyllostomidae muestran la mayor radiación de alimentación de todos los quirópteros (Howell 1974). Entre ellos se consideran especies carnívoras, piscívoras, insectívoras, frugívoras, hematófagas y nectarívoras (Altringham 1996). En América, los quirópteros que se alimentan de néctar pertenecen a la subfamilia Glossophaginae y son un componente frecuente de la fauna en las regiones tropicales y subtropicales. En México se distribuyen doce especies de glosofáginos y dos de ellas son endémicas (Arita \& Wilson 1987; Arita \& Santos del Prado 1999; Carstens et al. 2002; Tschapka \& Dressler 2002).

Los murciélagos nectarívoros presentan diversas modificaciones morfológicas como respuesta al tipo de dieta mientras que, al mismo tiempo, algunas plantas adaptaron sus estructuras florales para atraer a los quirópteros como polinizadores principales en un fenómeno denominado quiropterofília (Tschapka \& Dressler 2002; Waser 2005).

Los murciélagos nectarívoros generalmente presentan rostros alargados con lenguas largas y contráctiles provistas de papilas elongadas para acceder a la corola de la flor (Freeman 1995). Existen diferencias en la longitud craneal de cada especie, situación que se asocia con el grado de especialización en el consumo de néctar. En algunos glosofáginos, como Choeronycteris mexicana y Musonycteris harrisoni el alargamiento del rostro es muy conspicuo por lo que se cree que poseen una dieta líquida más especializada (Arita \& Martínez del Río 1990).

Se sabe que en general los murciélagos nectarívoros complementan la dieta con insectos y frutos. Las cantidades que consumen de estos alimentos varían en las diferentes especies de la subfamilia Glossophaginae y dependen en gran medida de su especialización morfológica (Heithaus 1982; Solmsen 1998). En este contexto, destaca el género Glossophaga que contiene especies con hocicos no muy alargados, dientes pequeños presentes en su totalidad y muelas desarrolladas para masticar alimento más consistente. Estos murciélagos, consumen en ciertas épocas grandes cantidades de frutas e insectos adicionales al néctar (Gardner 1977; Heithaus 1982; von Helversen 1993; Arita \& Santos del Prado 1999; Herrera et al. 2001; Arizmendi et al. 2002; Soriano \& Ruiz 2002).

En contraste, con base en análisis morfológicos y moleculares Anoura junto con seis géneros más (Choeronycteris, Choeroniscus, Hylonycteris, Lichonycteris, Musonycteris, y Scleronycteris), forma el clado Choeronycterines, referente a especies de murciélagos con alta especialización para el consumo de néctar (Carstens et al. 2002). Sin embargo y a pesar de sus adaptaciones, diversos autores reportan en Sudamérica que los murciélagos del género Anoura se alimentan de una gran 
cantidad de insectos y frutos (Sazima 1976; Gardner 1977; Willig et al. 1993; Nowak 1999; Zortéa 2003).

Anoura geoffroyi es una especie considerada nectarívora que se distribuye desde México hasta Sudamérica y que por sus hábitos alimentarios es un polinizador en los ecosistemas en los que se encuentra (Muchhala \& Jarrín-V 2002). No obstante que los estudios sobre este murciélago en México y en otros países son limitados, se sabe que en Brasil y Ecuador además de néctar, $A$. geoffroyi complementa su alimentación con frutos e insectos que al parecer no son consumidos por accidente (Nowak 1994; Muchhala \& Jarrín-V 2002). Al momento se ignora el tipo de insectos que consumen estos quirópteros (Muchhala \& Jarrín-V 2002).

Este trabajo tiene como objetivo describir los hábitos alimentarios de una población de Anoura geoffroyi, en particular la composición y la frecuencia con que consumen el alimento en Ixtapan del Oro, Estado de México, México.

\section{MATERIAL Y MÉTODOS}

Área de Estudio. El municipio de Ixtapan del Oro se encuentra en la parte Occidental del Estado de México, en la región montañosa que colinda con el estado de Michoacán. Su cabecera municipal se ubica a los $19^{\circ} 16^{\prime} 08^{\prime \prime}$ de latitud Norte y a los $100^{\circ} 16^{\prime} 52^{\prime}$ ' de longitud Oeste del meridiano de Greenwich. Se sitúa a una altitud de 1,705 m. Limita al Norte con el municipio de Donato Guerra, al Sur con Santo Tomás de los Plátanos, al Este con Valle de Bravo y al Oeste con el estado de Michoacán. El clima predominante es semicálido subhúmedo con lluvias en verano (A)c(W)a(i)g; el patrón de precipitación se encuentra entre los 1,000 y 1,300 mm, la escala isotérmica va de $\operatorname{los} 16$ a $20^{\circ} \mathrm{C}$. La topografía es muy accidentada y se caracteriza por elevadas montañas y pequeñas planicies, por lo que también, su vegetación es cambiante; al norte y en las partes altas se observa el Bosque de PinoEncino y al sur y en las partes bajas de la cañada predomina la Selva Baja Caducifolia de acuerdo con la clasificación de Miranda \& Hernández (1963). En el Bosque de Pino-Encino destacan en el estrato arbóreo Quercus sp., Juniperus flaccida, Pinus michoacana, Acacia sp., Dodonoea viscosa e Ipomoea murucoides. La Selva Baja Caducifolia tiene como dominantes en el estrato superior a los géneros Montanoa, Bursera, Acacia, Ipomoea y Ficus, y en el inferior Lupinus, Bouteloua, Acacia y Senecio (Gobierno del Estado de México 1980; Álvarez \& Álvarez-Castañeda 1996).

Captura de organismos. Las colectas se realizaron quincenalmente durante un periodo anual de Marzo de 2006 a Febrero de 2007. Se capturó a los murciélagos en un horario de las 21:00 a las 24:00 horas con una red de niebla de 2.5 X 6 m ubicada en la entrada de una mina situada a $2 \mathrm{~km}$ al Norte de la cabecera municipal de Ixtapan del Oro. Se clasificó a los organismos de acuerdo al sexo y considerando el grado de osificación en las epífisis de las falanges, en juveniles y adultos (Edythe 1988; Humphrey 1992; Ortega \& Arita 1999). Se determinó la condición reproductiva en 
las hembras; por el procedimiento de palpación abdominal directa se percibió si estaban preñadas u observando el desarrollo de los pezones, si eran lactantes (Humphrey 1992; Martino et al. 1998). Como indicador del tamaño se midió la longitud del antebrazo con un calibrador mecánico con $0.02 \mathrm{~mm}$ de precisión (Ceballos et al. 1997; Martino et al. 1998). El peso corporal se midió con una pesola de $100 \mathrm{~g}$ (+/- $1 \mathrm{~g}$ de precisión).

Se tomaron muestras de residuos del pelaje de las regiones cefálica, dorsal y ventral, de las extremidades posteriores y de las membranas alares mediante pequeños cuadros de gelatina de Kisser que fueron depositados dentro de viales plásticos. Antes de liberar a los murciélagos, se les depositó individualmente en bolsas de manta durante una hora para que evacuaran. Posteriormente, se revisó la bolsa para obtener las muestras de heces que se colocaron en viales plásticos (Beattie 1977; Herrera \& Martínez del Rio 1998; Muchhala \& Jarrín-V 2002).

Las muestras de los residuos del pelaje se montaron en laminillas y se les observó en un microscopio compuesto de campo claro a un aumento de 40X, en donde se identificaron y cuantificaron los morfotipos de polen y los restos de insectos (Herrera \& Martínez del Rio 1998; Muchhala \& Jarrín-V 2002). Las muestras de las heces fueron observadas en un microscopio de disección para ver si contenían semillas o pulpa de frutos. Posteriormente se les montó en laminillas con gelatina de Kisser y se les observó en un microscopio compuesto de campo claro a un aumento de 40X, en donde se identificaron y cuantificaron a los morfotipos de polen y restos de insectos (Kunz \& Whitaker 1983).

Para facilitar la identificación de las especies de las plantas correspondientes a los morfotipos de polen observados en las muestras; en el área de estudio se recolectó el polen de plantas que presentaban características del síndrome quiropterofílico para generar así una referencia palinológica y se realizó una comparación con las muestras obtenidas de Anoura geoffroyi. También, se utilizó literatura especializada en la determinación de plantas por medio del polen y se revisó bibliografía para la determinación del orden al que corresponden los restos de insectos (Whitaker 1988; McAney et al. 1991; Muchhala \& Jarrín-V 2002). Los granos de polen y los restos de insectos se contaron por la técnica de "escaneo" consistente en enfocarse en un campo visual y posteriormente desplazarse de forma horizontal a lo largo de toda la preparación para evitar contar repetidamente al mismo elemento (Girón 1995).

Análisis de datos. La cantidad de polen y de restos de insectos no son un parámetro para conocer en cuantas flores o insectos forrajean los murciélagos por lo que el análisis de los datos se basó en el cálculo de las frecuencias de ocurrencia (Muchhala \& Jarrín-V 2002). Cada muestra se evaluó como positiva en caso de presentar por lo menos un grano de polen o resto de insecto, y negativa en caso de no presentarlo y al final se sumaron las muestras positivas y se calculó su porcentaje respecto a la totalidad. Para observar posibles diferencias entre las frecuencias totales 
de los elementos y entre los sexos, se aplicaron pruebas de Chi-Cuadrada (Zar 1996; Muchhala \& Jarrín-V 2002). Se realizó la cuantificación de los granos de cada tipo de polen encontrado para calcular los índices de diversidad, equidad y dominancia de Simpson para cada mes de estudio (Girón 1995).

\section{RESULTADOS}

Se capturaron 153 murciélagos Anoura geoffroyi, con una proporción sexual de 2:1 con 96 machos y 57 hembras. Se obtuvieron datos de 145 adultos y 8 juveniles. Durante los meses de agosto y septiembre se capturaron 17 hembras preñadas y en diciembre y enero 2 hembras lactantes. También, en diciembre y enero se observó a 8 juveniles. El promedio $+/-\mathrm{S}$ del peso de los machos adultos fue de $16.83 \pm 1.49 \mathrm{~g}$ $(\mathrm{n}=92)$ y el de las hembras adultas no gestantes de $15.88 \pm 1.48 \mathrm{~g}(\mathrm{n}=36)$. El promedio general del peso de los adultos sin considerar a las 17 hembras grávidas fue de $16.57 \pm 1.54 \mathrm{~g}(\mathrm{n}=128)$ y el de los juveniles de $11.37 \pm 1.11 \mathrm{~g}(\mathrm{n}=8)$. El promedio $+/-\mathrm{S}$ de la longitud del antebrazo de los machos adultos fue de $43.93 \pm 1.30 \mathrm{~mm}$ $(\mathrm{n}=92)$ y el de las hembras adultas de $43.98 \pm 1.13 \mathrm{~mm}(\mathrm{n}=53)$. El promedio de la longitud del antebrazo de los adultos fue de $43.95 \pm 1.23 \mathrm{~mm}(\mathrm{n}=145)$ y el de los juveniles de $41 \pm 0 \mathrm{~mm}(\mathrm{n}=8)$.

Muestras del pelaje. Se obtuvieron 153 muestras de restos del pelaje en donde se encontraron polen y restos de insectos. 98 muestras resultaron positivas para granos de polen lo que representa el $64.04 \%$ del total y se obtuvieron 136 muestras positivas para restos de insectos que representan el $88.8 \%$ del total de las muestras. Existieron diferencias significativas entre las frecuencias de ambos componentes $\left(\mathrm{X}^{2}\right.$ $=26.22$; g. $1 .=1, \mathrm{P} \leq 0.05$ ).

De las 153 muestras 96 corresponden a machos y 57 a hembras. El 57\% de las muestras de los machos y el $75 \%$ de las muestras de las hembras resultaron positivas para granos de polen. Existieron diferencias significativas entre las frecuencias para ambos sexos $\left(\mathrm{X}^{2}=5.11 ; \mathrm{g} .1 .=1, \mathrm{P} \leq 0.025\right)$. Se observaron 8 morfotipos de polen de plantas que son polinizadas por murciélagos. Se obtuvo la mayor frecuencia de muestras positivas del polen correspondiente a los géneros Ipomoea, Agave y Pseudobombax. Las especies de Ipomoea que fueron colectadas, determinadas y comparadas con las muestras del pelaje y las heces fueron Ipomoea murucoides, I. lobata e I. purpurea. Sin embargo, no fue posible discriminar a que especie correspondía cada muestra, por lo que se consideró globalmente al género Ipomoea. Durante los meses de floración de Ipomoea murucoides (Octubre - Febrero), hubo una mayor frecuencia de polen de Ipomoea en las muestras del pelaje (Tabla 1).

La mayor riqueza de plantas visitadas por Anoura geoffroyi se registró en marzo con 7 morfotipos de polen, los meses con mayor diversidad fueron marzo y abril, el mes de mayor equidad fue abril y los de mayor dominancia fueron diciembre y enero en donde se observó la floración de Ipomoea murucoides en el sitio de estudio (Tabla 2). 
Caballero-Martínez et al. Hábitos alimentarios Anoura geoffroyi

Tabla 1. Frecuencias de las plantas observadas por el polen de las muestras del pelaje $(\mathrm{n}=153)$.

\begin{tabular}{lcc}
\hline Familia & Especie & Frecuencia \\
\hline Convolvulaceae & Ipomoea spp. & 48 \\
Agavaceae & Agave sp. & 36 \\
Bombacaceae & Pseudobombax ellipticum & 20 \\
Solanaceae & Brugmansia candida & 15 \\
Bombacaceae & Ceiba aesculifolia & 10 \\
Desconocida & & 10 \\
Asteraceae & & 8 \\
Cactaceae & Stenocereus sp. & 5 \\
\hline
\end{tabular}

Tabla 2. Índices de Simpson aplicados a los morfotipos de polen del pelaje por cada mes de estudio.

\begin{tabular}{lcccc}
\hline Mes & Riqueza $(\boldsymbol{S})$ & Diversidad $\left(\boldsymbol{d}_{\boldsymbol{s}}\right)$ Equidad $\left(\boldsymbol{e}_{\boldsymbol{s}}\right)$ & Dominancia $(\boldsymbol{\lambda})$ \\
\hline Marzo & 7 & 3.33 & 0.42 & 0.30 \\
Abril & 3 & 3.57 & 0.85 & 0.28 \\
Mayo & 3 & 3.00 & 0.33 & 0.33 \\
Junio & 4 & 2.00 & 0.35 & 0.50 \\
Julio & 5 & 1.16 & 0.23 & 0.85 \\
Agosto & 4 & 2.89 & 0.58 & 0.34 \\
Septiembre & 4 & 2.48 & 0.57 & 0.40 \\
Octubre & 5 & 1.89 & 0.32 & 0.52 \\
Noviembre & 2 & 1.08 & 0.51 & 0.92 \\
Diciembre & 2 & 1.00 & 0.50 & 0.99 \\
Enero & 3 & 1.00 & 0.33 & 0.99 \\
Febrero & 5 & 2.98 & 0.54 & 0.33 \\
\hline
\end{tabular}

El 95\% del total de los restos de insectos encontrados en las 153 muestras del pelaje correspondieron a restos de lepidópteros. El 86.45\% de las muestras de los machos y el $92 \%$ de las muestras de las hembras resultaron positivas para los restos de insectos. No existieron diferencias significativas entre las frecuencias para ambos $\operatorname{sexos}\left(\mathrm{X}^{2}=1.54 ;\right.$ g. $\left.1 .=1, \mathrm{P}>0.05\right)$.

Muestras de las heces. Se obtuvieron y analizaron un total de 61 excretas en las que se encontraron polen y restos de insectos. 60 muestras resultaron positivas para granos de polen lo que representa el $98.36 \%$ y se obtuvieron 56 muestras positivas para restos de insectos lo que representa el $91.8 \%$ del total de las muestras. No existieron diferencias significativas entre las frecuencias de ambos componentes $\left(\mathrm{X}^{2}\right.$ $=2.80 ;$ g. $1 .=1, \mathrm{P} \leq 0.10)$. 
De las 61 muestras de las heces 36 corresponden a machos y 25 a hembras. El $97.22 \%$ de las muestras de los machos y el $100 \%$ de las muestras de las hembras resultaron positivas para granos de polen. No existieron diferencias significativas entre las frecuencias para ambos sexos $\left(\mathrm{X}^{2}=0.70 ; \mathrm{g} .1 .=1, \mathrm{P} \leq 1\right)$. Se observaron 9 morfotipos de polen y se obtuvo la mayor frecuencia de muestras positivas en los morfotipos correspondientes a Ipomoea, Agave y Pseudobombax ellipticum. Durante los meses de floración de Ipomoea murucoides (Octubre - Febrero) hubo una mayor frecuencia de Ipomoea en las muestras de heces (Tabla 3).

Tabla 3. Frecuencias de las plantas determinadas por el polen de las muestras de heces $(n=61)$.

\begin{tabular}{lcc}
\hline Familia & Especie & Frecuencia \\
\hline Convolvulaceae & Ipomoea spp. & 59 \\
Agavaceae & Agave sp. & 38 \\
Bombacaceae & Pseudobombax ellipticum & 20 \\
Asteraceae & & 16 \\
Solanaceae & Brugmansia candida & 6 \\
Bombacaceae & Ceiba aesculifolia & 6 \\
Desconocida & & 6 \\
Mimosaceae & Calliandra sp. & 5 \\
Cactaceae & Stenocereus sp. & 4 \\
\hline
\end{tabular}

La mayor riqueza de plantas visitadas por Anoura geoffroyi se registró en marzo con 7 morfotipos de polen, la mayor diversidad se observó en marzo, abril y mayo, el mes con mayor equidad fue agosto y los de mayor dominancia fueron noviembre y febrero, meses en donde se observó la floración de Ipomoea murucoides en el sitio de estudio (Tabla 4).

Tabla 4. Índices de Simpson para los morfotipos de polen de las heces por cada mes de estudio.

\begin{tabular}{lcccc}
\hline Mes & Riqueza $(\boldsymbol{S})$ & Diversidad $\left(\boldsymbol{d}_{\boldsymbol{s}}\right)$ Equidad $\left(\boldsymbol{e}_{\boldsymbol{s}}\right)$ & Dominancia $(\boldsymbol{\lambda})$ \\
\hline Marzo & 7 & 2.67 & 0.30 & 0.37 \\
Abril & 5 & 2.52 & 0.44 & 0.39 \\
Mayo & 4 & 2.94 & 0.63 & 0.34 \\
Junio & 6 & 2.37 & 0.39 & 0.42 \\
Julio & 5 & 1.09 & 0.21 & 0.91 \\
Agosto & 2 & 2.50 & 10.40 & \\
Septiembre & 3 & 1.04 & 0.34 & 0.95 \\
Octubre & 3 & 1.23 & 0.60 & 0.81 \\
Noviembre & 2 & 1.02 & 0.25 & 0.97 \\
Diciembre & 4 & 1.33 & 0.33 & 0.75 \\
Enero & 6 & 1.64 & 0.27 & 0.60 \\
Febrero & 6 & 1.02 & 0.17 & 0.97 \\
\hline
\end{tabular}


E1 95\% del total de los restos de insectos encontrados en las 61 muestras de heces correspondieron a fragmentos y escamas de lepidópteros. El $88.88 \%$ de las muestras de los machos y el $96 \%$ de las muestras de las hembras resultaron positivas para los restos de insectos. No existieron diferencias significativas entre las frecuencias para ambos sexos $\left(\mathrm{X}^{2}=0.99 ;\right.$ g. 1. $\left.=1, \mathrm{P}<1\right)$.

\section{DISCUSIÓN}

En Ixtapan del Oro, Estado de México Anoura geoffroyi se alimenta de néctar, polen e insectos y no complementa su dieta con frutos como lo reportan para otros sitios Sazima 1976; Gardner 1977; Ceballos \& Galindo 1984 y Zortéa 2003. Por lo que no se le puede considerar en su totalidad como una especie frugívora. A diferencia de lo observado en Brasil por Zortéa (2003), quien menciona el consumo considerable de frutos por A. geoffroyi, Muchhala y Jarrín-V (2002) en su estudio del Bosque de Niebla del occidente de Ecuador no encontraron rastros de frutos en las muestras de la especie. Generalmente los ambientes con humedad constante no presentan una estacionalidad marcada en la disponibilidad de recursos vegetales, por lo que Muchhala y Jarrín-V (2002) concluyen que la dieta de A. geoffroyi no se complementa por frutos en el Bosque de Niebla debido a que es un ecosistema sin extremos climáticos drásticos en donde los murciélagos nectarívoros disponen de flores durante todos los meses del año. En el presente estudio realizado en la Selva Baja Caducifolia, ecosistema con marcada estacionalidad pero con una amplia disponibilidad de recursos (Rzedowski 1978; Trejo 1998), tampoco se encontraron semillas ni pulpa de frutos en las heces. Las muestras tuvieron una alta frecuencia de insectos ( $>90 \%$ ) y una alta cantidad de morfotipos de polen (9) cuya presencia, como se indica en las tablas 2 y 4 , se encuentra distribuida en todos los meses de estudio, por lo que $A$. geoffroyi puede alimentarse de néctar e insectos durante todo el año sin la necesidad de integrar frutos en su dieta.

Es complicado clasificar a los murciélagos en un gremio considerando sus hábitos alimentarios dado que un gran numero de especies altamente especializadas para el consumo de frutos o néctar consumen tanto néctar y frutos como insectos (Heithaus 1982; Pedro \& Taddei 1997; Muchhala \& Jarrín-V 2002; Zortéa 2003). No obstante, los resultados indican que Anoura geoffroyi en Ixtapan del Oro, presenta una alimentación basada en néctar, polen e insectos.

Si se considera que por lo menos el $95 \%$ de las partes de insectos encontradas corresponden a lepidópteros (artrópodos estrechamente asociados con las flores); (Sazima \& Sazima 1975; Mori \& Lovoba 1982; Silva \& Peracchi 1999; Lemus \& Ramírez 2003), es probable que $A$. geoffroyi consuma las mariposas nocturnas al momento de visitar las flores, por lo que de acuerdo con sus adaptaciones al consumo de néctar se podría catalogar a la población en estudio como murciélagos nectarívoros que tienen la capacidad de alimentarse de lepidópteros asociados a las 
flores que frecuenta. No obstante, son necesarios otros estudios que documenten si es un cazador activo de lepidópteros o los consume por oportunidad cuando están presentes en las flores.

Es probable que las necesidades alimentarias hayan conducido a que esta especie adaptada para consumir néctar adicione frecuentemente insectos a su dieta o bien, puede ser que nunca haya perdido este hábito. Por lo anterior, es necesario elucidar si el alto consumo de insectos observado en $A$. geoffroyi es un retroceso evolutivo (Solmsen 1998; Teotonio \& Michael 2000) o un comportamiento conservado de los ancestros insectívoros que se alimentaban de insectos asociados a las flores (Heithaus 1982).

Ocho de los nueve morfotipos de polen encontrados en las muestras, con excepción del polen desconocido, corresponden a plantas reportadas como parte de la dieta de murciélagos nectarívoros (Álvarez \& Gonzáles 1970; Ceballos \& Galindo 1984; Arita \& Wilson 1987; Eguiarte et al. 1987; Reid 1997; Riechers et al. 2003), y es el primer reporte de consumo de néctar de las plantas de la familia Asteraceae, de Brugmansia candida y de Stenocereus sp. para A. geoffroyi.

Los nueve morfotipos de polen revelan una baja fidelidad de $A$. geoffroyi por una planta en particular (Muchhala \& Jarrín-V 2002). No obstante, en las muestras del pelaje y heces se obtuvo una frecuencia mayor para el género Ipomoea. Estas plantas, incluyendo la especie Ipomoea murucoides, además de presentar algunas de las características típicas del síndrome quiropterofílico por tener flores de gran tamaño con producción de néctar y polen durante la noche (Oliveira \& Leitao-Filho 1987; Kinoshita et al. 2006), también son conocidas como parte esencial de la dieta de otras especies de murciélagos nectarívoros como Leptonycteris nivalis que consume Ipomoea durante su estancia invernal en Tepoztlán, Morelos (Sánchez 2004).

Los otros ocho morfotipos de polen identificados en las muestras de $A$. geoffroyi corresponden a plantas que también son parte de la dieta de varias especies de murciélagos nectarívoros (Arita \& Wilson 1987; Eguiarte et al. 1987; Riechers et al. 2003; Sperr 2004). Las especies del genero Agave mantienen una relación tan estrecha con los murciélagos que son casi exclusivamente polinizadas por quirópteros de la subfamilia Glossophaginae (Arita \& Wilson 1987). Entre las adaptaciones más notables de estas plantas, están la presencia de flores que sólo abren al anochecer y la producción de néctar y polen exclusivamente nocturna (Arita \& Wilson 1987; Arita \& Santos del Prado 1999).

Las flores de Pseudobombax ellipticum producen néctar abundante durante la noche. En Morelos son visitadas por murciélagos nectarívoros de las especies Choeronycteris mexicana, Glossophaga leachii y en Chiapas por Leptonycteris curasoae (Eguiarte et al. 1987; Sánchez-Casas \& Álvarez 2000; Riechers et al. 2003). No obstante, su grado de especialización con el polinizador no es tan estrecho como en los agaves ya que durante el día esta planta también es visitada por otros polinizadores como aves e insectos y en las noches por polillas (Eguiarte et al. 1987). 
Las especies de la familia Asteraceae presentan inflorescencias con características entomófilas (Proctor et al. 1996), sin embargo como lo mencionan Sánchez y AmatGarcía (2005) la mayoría de los insectos que las visitan no son considerados polinizadores importantes, por lo que algunas especies de estas plantas atraen a otros animales que pudieran polinizarlas (Proctor et al. 1996). Sánchez-Casas y Álvarez (2000) y Riechers et al. (2003) en sus trabajos realizados con Leptonycteris curasoae y varias especies del género Glossophaga describen que estos murciélagos se alimentan del néctar de plantas pertenecientes a la familia Asteraceae.

Brugmansia candida cuya sinonimia es Datura candida, es una planta nativa de Sudamérica que se extendió hacia todas las regiones tropicales del continente Americano, tiene flores de gran tamaño de 20 a $25 \mathrm{~cm}$ de longitud con coloraciones pálidas y producen néctar y aroma durante la noche (Brako \& Zarucchi 1993; Chavarría et al. 2000). Las flores de esta especie son generalmente atractivas para los insectos, especialmente los lepidópteros (Iqbal et al. 2001). Al ser una planta que atrae polillas en abundancia por su olor (Harbone 1995), es probable que Anoura geoffroyi la visite no sólo para consumir el néctar sino también para capturar lepidópteros.

Las flores de Ceiba aesculifolia presentan características del síndrome quiropterofílico ya que son de color pálido de pardo a blanco y producen néctar durante la noche (Carranza \& Blanco 2000), son visitadas por un gran número de especies de murciélagos nectarívoros como Anoura geoffroyi, Glossophaga soricina y Leptonycteris curasoae (Riechers et al. 2003; Quesada et al. 2004; Sperr 2004).

Dado que producen néctar durante la noche, varias especies del género Calliandra forman parte de la dieta de Glossophaga soricina y Leptonycteris curasoae (Lemke 1985; Riechers et al. 2003; Hernández-Conrique et al. 2007). Sin embargo, estas plantas no son exclusivamente polinizadas por murciélagos. Durante el día también producen néctar y son frecuentadas por insectos y aves que, al parecer, son polinizadores poco eficaces para estas plantas (Hernández-Conrique et al. 2007).

Las flores del género Stenocereus se adaptaron de tal forma que principalmente aceptan a polinizadores específicos como los murciélagos del género Leptonycteris, sin embargo, en menor proporción, también pueden ser polinizadas por insectos y aves (Ceballos et al. 1997; Fleming \& Nassar 2002; Valiente-Banuet 2002; ArreolaNava \& Terrazas 2003, Ibarra-Cerdeña et al. 2005). En las cactáceas columnares de los desiertos del Norte de México Ceballos et al. (1997) encontraron que por las distancias en que se encuentran las plantas sería casi imposible que fueran polinizadas por otro agente que no sean los quirópteros que pueden recorrer trayectos muy largos cada noche.

Por lo tanto, casi todas las plantas que se determinaron por los morfotipos de polen presentan algunas de las características descritas para el síndrome quiropterofílico entre las que destaca la producción de polen y néctar durante la 
noche (Sazima \& Sazima 1975; Mori \& Lovoba 1982; Silva \& Peracchi 1999; McDonald 2001; Tschapka \& Dressler 2002; Waser 2005). Sin embargo, gran parte de estas plantas también concuerdan con algunas de las características del síndrome entomófilo; en particular las de la familia Asteraceae, Brugmansia candida, Calliandra, Ipomoea y Pseudobombax ellipticum (Eguiarte et al. 1987; Harbone 1995; Proctor et al. 1996; Iqbal et al. 2001; Arreola-Nava \& Terrazas 2003; Hernández-Conrique et al. 2007), por lo que es probable que Anoura geoffroyi también seleccione a estas flores para capturar insectos.

La observación de hembras lactantes coincidió en el mes de diciembre con la floración de Ipomoea murucoides en el sitio de estudio, cuyo polen se presentó con mayor frecuencia en las muestras por lo que es probable que $A$. geoffroyi sincronice su ciclo reproductivo con los meses de mayor abundancia de Ipomoea. Esta característica se observó en otros murciélagos nectarívoros como Glossophaga soricina y Leptonycteris curasoae (Racey 1982; Baumgarten \& Vieira 1994; Fleming \& Nassar 2002; Mena \& Williams 2002; Zortéa 2003). La sincronización de la lactancia con el tiempo de floración debe ayudar a las hembras a sobrevivir durante el momento reproductivo de mayor demanda energética (Racey 1982; Dinerstein 1986; Heideman et al. 1992; Singh \& Krishna 1997; Heideman \& Powell 1998; Porter \& Wilkinson 2000).

En las muestras del pelaje, las hembras presentaron una frecuencia significativamente mayor que los machos en la presencia de granos de polen. Sin embargo, estas diferencias no se observaron en las muestras de las heces. En diversas especies de murciélagos de zonas templadas y tropicales las hembras necesitan una dieta rica en nutrientes durante la gestación y especialmente en la lactancia (Racey 1982; Singh \& Krishna 1997; Heideman \& Powell 1998; Porter \& Wilkinson 2000), por lo que es factible que las discrepancias sexuales de las frecuencias de granos de polen en las muestras del pelaje se deban a que por necesidades reproductivas las hembras visiten con mayor frecuencia a las flores para alimentarse del néctar o probablemente se acerquen a ellas en busca de insectos.

Los índices de diversidad en las tablas 2 y 4 muestran que aunque en todos los meses existen flores disponibles para $A$. geoffroyi se modifica el número de especies de la dieta y se incrementa la dominancia de alguna de ellas (disminuye la diversidad al incrementar la floración de Ipomoea murucoides). Esta disponibilidad anual de alimento en forma de néctar y polen posiblemente sea la razón por la cual la población de $A$. geoffroyi permanece durante todo el año en el mismo sitio. Aunque también, existe la posibilidad de que algunos individuos realicen movimientos estacionales siguiendo gradientes de floración como las especies del género Leptonycteris (Fleming \& Nassar 2002).

La especie Anoura geoffroyi no solo tiene una gran relevancia ecológica como polinizador y controlador de las poblaciones de insectos, sino que también como 
polinizador es de gran importancia para el hombre ya que algunas de las plantas que visita tienen alguna utilidad como los agaves para preparar bebidas, Brugmansia candida es de uso ornamental y las cactáceas son ornamentales y producen frutos comestibles.

AGRADECIMIENTOS. Agradecemos al Consejo Nacional de Ciencia y Tecnología (CONACyT) y al Consejo Mexiquense de Ciencia y Tecnología (COMECyT) por el aporte de los recursos económicos y a la Facultad de Ciencias de la Universidad Autónoma del Estado de México por facilitar parte del equipo para la realización del estudio. También a Virginie Michou Ferrara por su ayuda durante todo el estudio de campo y al herbario de la Facultad de Ciencias de la UAEMéx por la determinación taxonómica de las plantas.

\section{LITERATURA CITADA}

Altringham, J. D. 1996. Bats: biology and behaviour. Oxford University Press, Oxford, Inglaterra.

Álvarez, T. \& S. Álvarez-Castañeda. 1996. Aspectos biológicos y ecológicos de los murciélagos de Ixtapan del Oro, México. Pp. 169-182. In: Contribution in Mammalogy: A Memorial volume Honoring Dr. J. Knox Jones. Jr. Museum Texas, Estados Unidos de Norteamérica.

Álvarez, T. \& L. Gonzáles. 1970. Análisis polínico del contenido gástrico de murciélagos Glossophaginae de México. Anales de la Escuela Nacional de Ciencias Biológicas. 18:137-165.

Arita, H. T. \& D. Wilson. 1987. Long-nosed bats and agaves: The tequila connection: The interdependence between bats and agaves is so strong that one might not be able to survive without the other. Bats 4: 3-5.

Arita, H. T. \& C. Martínez del Río. 1990. Interacciones flor-murciélago: Un enfoque zoocentrico. Instituto de Biología (UNAM). D.F., México.

Arita, H. T. \& K. Santos del Prado. 1999. Conservation biology of the nectar-feeding bats in Mexico. Journal of Mammalogy 80: 31-41.

Arreola-Nava, H. J. \& T. Terrazas. 2003. Especies de Stenocereus con aréolas morenas: Clave y descripciones. Acta Botánica Mexicana 64: 1-18.

Arizmendi, M., A. Valiente-Banuet, A. Rojas-Martínez, \& P. Dávila-Aranda. 2002. Columnar cacti and the diets of nectar-feeding bats. Pp. 264-282. In: T. Fleming \& A. Valiente-Banuet (eds) Columnar cacti and their mutualists. The University of Arizona Press, Tucson.

Baumgarten, J. \& E. Vieira. 1994. Reproductive seasonality and development of Anoura geoffroyi (Chiroptera: Phyllostomidae) in central Brazil. Mammalia 58(3): 415-422.

Beattie, A. J. 1971. A technique for the study of insect-borne pollen. Pan-Pacific Entomologist 47: 82.

Brako, L. \& J. L. Zarucchi. 1993. Catalogue of the flowering plants and gymnosperms of Peru. Monographs in Systematic Botany from the Missouri Botanical Garden. Missouri.

Carstens, B., B. Lundrigan, \& P. Myers. 2002. A phylogeny of the neotropical nectar-feeding bats (Chiroptera: Phyllostomidae) based on morphological and molecular data. Journal of Mammalian Evolution 9: 23-53.

Ceballos, G. \& C. Galindo. 1984. Mamiferos Silvestres de la Cuenca de México. Limusa-Instituto de Ecología. D.F., México.

Ceballos, G., T. Fleming, C. Chávez, \& J. Nassar. 1997. Population dynamics of Leptonycteris curasoae (Chiroptera: Phyllostomidae) in Jalisco, Mexico. Journal of Mammalogy 8: 1220-1230.

Chavarría, F., R. Espinoza, A. Guadamuz, D. Perez \& A. Masís. 2000. Species: Brugmansia candida (Solanaceae). Área de Conservación de Guanacaste. San José, Costa Rica. 
Dinerstein, E. 1986. Reproductive ecology of fruit bats and the seasonality of fruit production in a Costa Rica cloud forest. Biotropica 18(4):307-318.

Edythe, L. P. 1988. Age determination in bats. Pp. 47-58. In T. H. Kunz (ed) Ecological and behavioral methods for the study of bats. Washington, DC. Smithsonian Institution Press. Washington D.C.

Eguiarte, L., C. Martinez del Rio \& H. Arita. 1987. El néctar y el polen como recursos: El papel ecológico de los visitantes a las flores de Pseudobombax ellipticum (H.B.K.) Dugand. Biotropica 19: 74-82.

Fleming, T. H. \& J. Nassar. 2002. Population biology of the lesser long-nosed bat Leptonycteris curasoaein Mexico and northern South America. Pp. 283-305. In T.H. Fleming \& A. Valiente-Banuet (eds) Columnar cacti and their mutualists: Evolution, ecology, and conservation. Tucson, Arizona. The University of Arizona Press. Arizona.

Freeman, P. W. 1995. Nectarivorous feeding mechanisms in bats. Biological Journal of the Linnean Society 56: 439-463.

Gardner, A. L. 1977. Feeding habits. Pp. 293-350. In: R. Barker, J. Jones \& D. Carter (eds) Biology of bats of the New World family Phyllostomidae, Part II . Special Publications, The Museum of Texas Tech University.

Girón, M. V. 1995. Análisis palinológico de la miel y la carga de polen colectada por Apis mellifera en el suroeste de Antioquia, Colombia. Boletín del Museo de Entomología de la Universidad del Valle 3: $35-54$.

Gobierno del Estado de México. 1980. Monografía Municipal de Ixtapan del Oro. Estado de México, México.

Harbone, J. B. 1995. Recent advances in chemical ecology. Plant Science Laboratories, University of Reading. Londres, Inglaterra.

Heideman, P. D. \& F. H. Bronson. 1994. An endogenous circannual rhythm of reproduction in a tropical bat, Anoura geoffroyi, is not entrained by photoperiod. Biology of Reproduction 50: 607-614.

Heideman, P. D. \& K. S. Powell. 1998. Age-specific reproductive strategies and delayed embryo development in an old world fruit bat, Ptenochirus jagori. Journal of Mammalogy 79: 295-311.

Heithaus, R. E. 1982. Coevolution between bats and plants. Pp. 327-367. In: T. Kunz (ed) Ecology of Bats. Plenum Press. Nueva York.

Hernández-Conrique, D., J. F. Ornelas, J. G. García \& C. F. Vargas. 2007. Nectarproduction of Calliandra longipedicellata (Fabaceae: Mimosoideae), an endemic mexican shrub with multiple potential pollinators. Biotrópica 1- 9 .

Herrera, G. \& C. Martínez del Rio. 1998. Pollen digestion by new world bats: effects of processing time and feeding habits. Ecology 79: 2828-2838.

Herrera, G., K. Hobson, L. Mirón, N. Ramírez, G. Méndez \& V. Sánchez-Cordero. 2001. Sources of protein in two species of phytophagous bats in a seasonal dry forest: evidence from stable-isotope analysis. Journal of Mammalogy 82: 352-361.

Howell, D.J. 1974. Food habits of some Costa Rican bats. Revista Biología Tropical 21: 281-294.

Humphrey, S.R. 1992. Bats: Vespertilionidae and Molossidae. Pp. 52-67. In A. Chapman. \& A. Feldhamer (eds) Wild mammals of North America (Biology, management and economics). The Johns Hopkins University Press. Baltimore.

Ibarra-Cerdeña C., L. Iñiguez-Dávalos \& V. Sánchez-Cordero. 2005. Pollination ecology of Stenocereus queretaroensis (Cactaceae), a chiropterophilous columnar cactus, in a tropical dry forest of Mexico. American Journal of Botany 92: 503-509.

Iqbal, M., K. Wijesekera \& R. Hapukotuwa. 2001. Fruits and flowers in Brugmansia candida. Ceylon Journal of Science, Biological Sciences 28: 19-20. 
Kinoshita, L. S, R. Torres, E. Forni-Martins, J. Tamashiro, T. Spinelli, Y. Ahn \& S. Constancio. 2006. Composição florística e síndromes de polinização e de dispersão da mata do Sítio São Francisco, Campinas, SP, Brasil. Acta Botanica Brasilica 20: 313-327.

Kunz, T. H. \& J. O. Whitaker. 1983. An evaluation of fecal analysis for determining food habits of insectivorous bats. Canadian Journal of Zoology 61: 1317-1321.

Lemke, T. O. 1985. Pollen carrying by the nectar-feeding bat Glossophaga soricina in a suburban environment. Biotropica 17: 107-111.

Lemus, L. J. \& N. Ramírez. 2003. Polinización y polinizadores en la vegetación de la planicie costera de Paraguana, estado Falcon, Venezuela. Acta Científica Venezolana 54: 97-114.

Martino, A., A. Arends \& J. Aranguren. 1998. Reproductive pattern of Leptonycteris curasoae miller (Chiroptera: Phyllostomidae) in northern Venezuela. Mammalia 62: 69-76.

McAney, C. M., C. Shiel, C. Sulliven \& J. Fairley. 1991. The analysis of bat droppings. Occasional Publications of the Mammal Society 14: 1-48.

McDonald, A. 2001. Revision of Ipomoea series Tyrianthinae (Convolvulaceae). Lundellia 4: 76-93.

Medellín, R., H. Arita \& O. Sánchez. 1997. Identificación de los murciélagos de México: Clave de campo. Asociación Mexicana de Mastozoología. D.F., México.

Mena, J.L. \& M. Williams. 2002. Diversidad y patrones reproductivos de quirópteros en un área urbana de Lima, Perú. Ecología Aplicada 1: 1-9.

Miranda, F. \& E. Hernández X. 1963. Los tipos de vegetación de México y su clasificación. Boletín de la Sociedad Botánica de México 28: 29-179.

Mori, S. A. \& T. A. Lovoba. 1982. Bat/plant interactions in the Neotropics. The New York Botanical Garden. Comunicado especial. Nueva, York.

Muchhala, N. \& P. Jarrín-V. 2002. Flower visitation by bats in cloud forests of Western Ecuador. Biotropica 34: 387-395.

Nowak, R.M. 1994. Walker's Bats of the World. The Johns Hopkins University Press. Baltimore.

Nowak, R. 1999. Walker's Mammals of the World: Sixth Edition; Volume 1. The John Hopkins University Press. Baltimore.

Oliveira, P. S. \& H. Leitao-Filho. 1987. Extrafloral nectaries: Their taxonomic distribution and abundance in the woody flora of Cerrado vegetation in Southeast Brazil. Biotropica 19: 140-148.

Ortega, J. R. \& T. H. Arita. 1999. Structure and social dynamics of harem groups in Artibeus jamaicensis (CHIROPTERA: PHYLLOSTOMIDAE). Journal of Mammalogy 80: 117-1185.

Pedro, W. A. \& V. A. Taddei. 1997. Taxonomic assemblage of bats from Panga Reserve, south Brazil: Abundance and trophic relations in the Phyllostomidae (Chiroptera). Boletim do Museu de Biologia Mello 6:3-21.

Porter, T. A. \& G. S. Wilkinson. 2000. Birth synchrony in greater spear-nosed bats (Phyllostomus hastatus). Journal of Zoology 253: 383-390.

Proctor, M., P. Yeo \& A. Lack. 1996. The Natural History of pollination. Harper Collins. Londres, Inglaterra.

Quesada, M., et al. 2004. Effects of forest fragmentation on pollinator activity and consequences for plant reproductive success and mating patterns in bat-pollinated Bombacaceous trees. Biotropica 36: 131-138.

Racey, P. A. 1982. Ecology of bat reproduction. Pp. 169-171. In T. Kunz (ed) Ecology of bats. Plenum Press. New York.

Reid, F. 1997. A field guide to the mammals of Central America and Southeast Mexico. Oxford University Press. Oxford, Inglaterra.

Riechers, A. P., M. Martínez-Coronel \& R.L. Vidal. 2003. Consumo de polen de una colonia de maternidad de Leptonycteris curasoae yerbabuenae en Tuxtla Gutiérrez, Chiapas, México. Anales del Instituto de Biología UNAM 74: 43-66. 
Rzedowski, J. 1978. Vegetación de México. Limusa. D.F., México.

Sánchez, T. L. 2004. Dieta del murciélago magueyero mayor Leptonycteris nivalis (Chiroptera: Phyllostomidae) en la Cueva del Diablo, Tepoztlán, Morelos. Tesis de Biología. Facultad de Ciencias, UAEM. México, México.

Sánchez, N. D. \& G. D. Amat-García. 2005. Diversidad de la fauna de artrópodos terrestres en el humedal Jaboque, Bogotá - Colombia. Caldasia 27: 311-329.

Sánchez-Casas, N. \& T. Álvarez. 2000. Palinofagia de los murciélagos del género Glossophaga (Mammalia: Chiroptera) en México. Acta Zoológica Mexicana 81: 23-62.

Sazima, I. 1976. Observations on the feeding habits of Phyllostomid bats (Carollia, Anoura and Vampyrops) in Southeastern Brazil. Journal of Mammalogy 57: 381-382.

Sazima, I. \& M. Sazima. 1975. Polinização das flores pelos morcegos. Só Brasil. Editora ABZ Ltda. São Paulo. Brasil.

Singh, U. P. \& V. A. Krishna. 1997. A mini review on the female Chiropteran reproduction. Journal of Endocrinal Reproduction 1: 1-19.

Silva, S. S. \& A. L. Peracchi. 1999. Visits of bats to flowers of Lafoensia glyptocarpa Koehne (Lythraceae). Revista Brasileira de Biologia 59: 19-22.

Solmsen, E. H. 1998. New World nectar-feeding bats: biology, morphology and craniometric approach to systematics. Bonner zoologische Monographien 44: 1-118.

Soriano, P. J. \& A. Ruiz. 2002. The role of bats and birds in the reproduction of columnar cacti in the Northern Andes. Pp. 241-263. In: T. Fleming \& A. Valiente-Banuet (eds) Columnar cacti and their mutualists. The University of Arizona Press. Tucson.

Sperr, E. B. 2004. Blütenbesucher im Trockenwald: Nahrungswahl und Ökologie der hochspezialisierten Blumenfledermaus Musonycteris harrisoni und koexistierender Arten. Diploma-Thesis, University of Ulm. Ulm, Alemania.

Teotonio, H \& R. Michael. 2000. Perpective: Reverse evolution. Evolution 55: 653-660.

Trejo, I. 1998. Distribución y diversidad de selvas bajas de México: relaciones con el clima y el suelo. Tesis Doctoral Facultad de Ciencias, UNAM. D.F., México.

Tschapka, M. \& S. Dressler. 2002. Chiropterophily: on bat-flowers and flower bats. Royal Botanic Gardens 114-125.

Valiente-Banuet, A. 2002. Vulnerabilidad de los sistemas de polinización de cactáceas columnares de México. Revista Chilena de Historia Natural 75: 99-104.

von Helversen, O. 1993. Adaptations of flowers to the pollination by glossophagine bats. Pp. 41-59. In: W. Barthlott, C. Naumann, K. Schmidt-Loske K. \& K. Schuchman (eds) Animal-plant interactions in tropical environment. Museum Koenig. Bon, Alemania.

Waser, N. M. 2005. Specialization and generalization in plant-pollinator interactions: A $\mathrm{h}$ is t o r ic a 1 perspective. Pp. 3-17. In: N. Waser \& J. Ollerton (eds.) Plant-Pollinator interactions. The University of Chicago press. Chicago.

Whitaker, J. O. 1988. Food habits analysis of insectivorous bats. Pp. 171-189. In: T. Kunz (ed) Ecological and behavioral methods for the study of bats. Smithsonian Institution Press. Washington, D.C.

Willig, M., G. Camilo \& S. Noble. 1993. Dietary overlap in frugivorous and insectivorous bats from edaphic cerrado habitats of Brazil. Journal of Mammology 74: 117-128.

Zar, J. H. 1996. Biostatistical Analysis. 3a. edición, Prentice Hall. New Jersey.

Zortéa, M. 2003. Reproductive patterns and feeding habits of three nectarivorous bats (Phyllostomidae: Glossophaginae) from the Brazilian Cerrado. Brazilian Journal of Biology 63: 159-168. 